Proc. Indian Acad. Sci. (Earth Planet. Sci.), Vol. 95, No. 2, July 1986, pp. 265-273.

(C) Printed in India.

\title{
The effect of whitecaps and foam on wind speed extraction with a pulse limited radar altimeter
}

\author{
R M GAIROLA and P C PANDEY \\ Meteorology and Oceanography Division, Space Applications Centre, Ahmedabad \\ 380053, India
}

MS received 29 August 1985; revised 21 November 1985

\begin{abstract}
The effect of whitecaps and foam on wind speed extraction with a pulse limited radar altimeter has been studied using a specular point model. By modelling foam and water as a two-layer media, we have investigated the changes in reflectivity as a function of thickness using electro-magnetic field theory. Our analysis indicated a change of reflectivity from a value of 0.617 at $13.9 \mathrm{GHz}$ normal incidence to a value of 0.10 for a foam thickness of less than $1 \mathrm{~cm}$. The values of reflectivity computed from a two-layer model compared fairly well with these derived using an emissivity model.

The modified specular point model gave an improved relationship between $\sigma^{\circ}$ and wind speed in comparison to Brown's model. The Seasat altimeter's data analysis over the Arabian sea and the Bay of Bengal gave an rms difference of $2.2 \mathrm{~m} / \mathrm{sec}$ in wind speed retrieval using the present modified model and Brown's model.
\end{abstract}

Keywords. Radar altimeter; Seasat; wind speed; specular point model; two-layer media.

\section{Introduction}

Measurement of the wind field is important for several meteorological and oceanographic studies, e.g. Moisture transport, upwelling phenomena, sea-state and air-sea interaction study etc. Major oceanic currents are, in fact, driven by wind stress. For a tropical country like India, where geostrophic assumption breaks down, measurement of wind speed assumes even greater significance. In situ ship observations are inadequate and can take several days to cover a tiny portion of the ocean. Satellites, therefore, offer the best means of covering the vast data sparse oceanic regions.

Both active and passive microwave sensors have been used for wind speed determination. The Seasat satellite launched by NASA in June 1978 and managed by the Jet Propulsion Laboratory, USA, carried onboard an SMMR (scanning multichannel microwave radiometer), a radar altimeter, a scatterometer and a synthetic aperture radar. The SMMR, altimeter and scatterometer data have been used to determine wind speeds and a global comparison of wind speeds have been made by Pandey (1983). The scatterometer, in addition, provides wind direction. It was specifically designed for wind vector measurement with a wide swath coverage $(500 \mathrm{~km})$. The altimeter, on the other hand, provides along the track wind field data most useful for climatological studies. The primary objective of the radar altimeter is to provide significant wave height and sea surface topography. Also, the nadir wind speed together with significant wave height could be used for estimating swell amplitude (or energy) from the radar altimeter (Mognard 1984). The forthcoming mission of the European Space Agency, ERS-1 scheduled for launch in 1989 , will also carry a radar altimeter, similar to the Seasat altimeter. 
For a radar altimeter, the backscattering coefficient, $\sigma^{\circ}$ is dominated by specular reflection and is proportional to the Fresnel reflection coefficient and inversely proportional to the mean square slope (Brown 1978). For a high sea state, foam and whitecaps appear due to breaking of waves. The waves break when the condition, that water particles speed exceed the average speed of the waves in the ocean, is satisfied. Foam and whitecaps in the ocean surface have electromagnetic properties different from the background sea water. Air, foam and sea water form a layered media, distort the fine structure of the surface and alter reflectivity due to resonant phenomenon. We present the investigation of this phenomena, following Wait (1962) and Zheng et al (1983), using electromagnetic field theory and its impact on wind speed extraction from a radar altimeter. In our study we have used a frequency dependent fractional foam coverage and an empirical emissivity model proposed by Pandey and Kakar (1982), and Hollinger's (1973) formulations for the Debye parameters.

\section{Specular point model}

In the interaction of microwaves with a target such as the surface of the sea in which a large area is illuminated, the average returned power $P_{r}(t)$ can be expressed by the radar equation

$$
P_{r}(t)=\frac{\lambda^{2}}{(4 \pi)^{3}} \int_{\text {illuminated area }} \frac{P_{t}(t) G_{t}^{2} \sigma^{\circ}}{R^{4}} \mathrm{~d} A,
$$

where

$P_{r}=$ the average received power (in watts), $P_{t}=$ the transmitted power (in Watts), $G_{t}=$ the antenna gain, $\lambda=$ the operating wavelength, $R=$ the range to the target and $\sigma^{\circ}=$ the scattering cross-section per unit area $\left(\mathrm{W} / \mathrm{m}^{2}\right)$.

The average normalised backscattering cross-section is a convenient measure used to describe the reflectivity of an extended target or surface. For a radar altimeter, at a frequency of about $14 \mathrm{GHz}$ and $0^{\circ}$ incidence angle, the average power scattered by the sea surface is proportional to the average number of specular points per unit area and to the average Gaussian and isotropic curvature of the specular points. These two parameters have been related to surface statistics through geometric optics considerations and result in the following expression for average scattering cross-section (Barrick 1974).

$$
\sigma^{\circ}(\theta)=\pi|R(\theta)|^{2} \sec ^{4} \theta p\left(\zeta_{x s p}, \zeta_{y s p}\right)
$$

where

$$
\begin{aligned}
& \theta=\text { incidence angle, } \\
& p\left(\zeta_{x s p}, \zeta_{y s p}\right)=\text { surface slope probability density function, (p.d.f.) } \\
& \zeta_{x}=\frac{\partial n}{\partial x}, \zeta_{y}=\frac{\partial n}{\partial y}, n=\text { surface elevation, }
\end{aligned}
$$

$R(\theta)=$ the Fresnel reflection coefficient of air to surface interface at incidence angle $(\theta)$. 
Under the classical assumption when the sea surface slopes are nearly Gaussian and isotropic in their distribution, the p.d.f. is given by

$$
p\left(\zeta_{x}, \zeta_{y}\right)=\frac{1}{\pi S^{2}} \exp \left(-\tan ^{2}(\theta) / S^{2}\right)
$$

$\bar{S}^{2}$, the mean square slope, is given by

$$
\bar{S}^{2}=\left\langle\zeta_{x}^{2}+\zeta_{y}^{2}\right\rangle
$$

and the scattering coefficient is given by

$$
\sigma^{\circ}(\theta)=\frac{|R(\theta)|^{2}}{S^{2}} \sec (\theta) \exp \left(-\tan ^{2}(\theta) / S^{2}\right)
$$

The expression (6) for $\sigma^{\circ}(\theta)$ is valid for $(\theta) \leqslant 15^{\circ}$ and under the condition that the curvature of the surface waves is much larger than the wavelength of the incident electromagnetic wave (Valenzuela 1978). Different forms of dependence of $S^{2}$ on the wind speed are available, the classical one being the work of Cox and Munk (1954).

\section{Reflection from two-layer fosm/water media}

The general analysis of reflection of plane waves from a parallel stratified medium consisting of $M$ homogeneous slabs has been described by Wait (1962) and a two-layer case by Zheng et al (1983). Considering the case of a two-layer medium (figure 1) and normal incidence with the interface of air, foam and water where the conductivity, permeability and dielectric constant of each layer is denoted by $\sigma_{i}, \mu_{i}, \varepsilon_{i}(i=0,1,2)$, the reflection coefficient is given by:

total reflection coefficient $=\frac{b_{0}}{a_{0}}=\frac{k_{0}-k_{1} Q}{k_{0}+k_{1} Q}$

and reflectivity, $r f=\left|\frac{b_{0}}{a_{0}}\right|^{2}$ under the condition $r_{1} / r_{0} \gg 0, r_{2} / r_{0} \gg 0$ $h_{2} \sim \infty$,

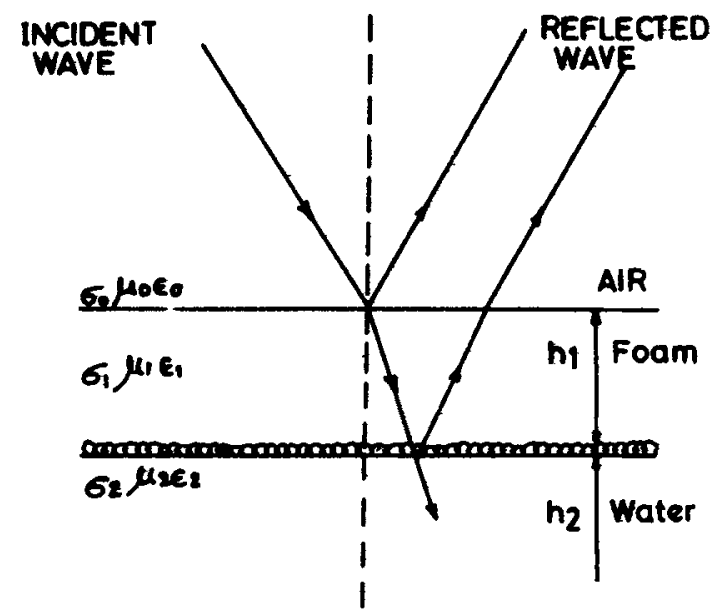

Figure 1. Reflection, propagation and transmission of electromagnetic waves in a stratified medium consisting of air, foam and water. 
where $Q$ is the correction to the characteristic impedence $k_{1}$ of the upper layer to account for the presence of the lower layer. The quantities $k_{0}, k_{2}$ and $Q$ are given by

$$
\begin{aligned}
& k_{0}=\left(\mu_{0} / \varepsilon_{0}\right)^{1 / 2}, \\
& k_{1}=r_{1} /\left(\sigma_{1}+i \varepsilon_{1} \omega\right), \\
& Q=\frac{r_{1} / r_{2}+\tanh \left(r_{1} h_{1}\right)}{\left[1+\left(r_{1} / r_{2}\right) \tanh \left(r_{1} h_{1}\right)\right]} .
\end{aligned}
$$

The propagation constants $r_{1}, r_{2}$ are given by

$$
r_{m}=\left[i \sigma_{m} \mu_{m} \omega-\varepsilon_{m} \mu_{m} \omega^{2}\right]^{1 / 2}, m=1,2
$$

and

$$
\begin{aligned}
\varepsilon_{i} & =\varepsilon_{0} \varepsilon_{i}^{\prime}, \\
\sigma_{i} & =\omega \varepsilon_{0} \varepsilon_{i}^{\prime \prime}, \\
\varepsilon_{i}^{r} & =\varepsilon_{i}^{\prime}-i \varepsilon_{i}^{\prime \prime},
\end{aligned}
$$

and

$$
C=\left(\mu_{0} \varepsilon_{0}\right)^{1 / 2},
$$

where $\varepsilon_{i}$ is called the complex dielectric constant of the medium and $\varepsilon_{0}$ is the dielectric constant of the vacuum rewriting (9) and (11).

$$
\begin{aligned}
& k_{1}=r_{1} / \omega \varepsilon_{0}\left(\varepsilon_{1}^{\prime \prime}+i \varepsilon_{1}^{\prime}\right), \\
& r_{1}=(2 \pi / \lambda)\left(i \varepsilon_{1}^{\prime \prime}-\varepsilon_{1}^{\prime}\right)^{1 / 2}, \\
& r_{2}=(2 \pi / \lambda)\left(i \varepsilon_{2}^{\prime \prime}-\varepsilon_{2}^{\prime}\right)^{1 / 2},
\end{aligned}
$$

where $\lambda$ is the free space wavelength

$$
\frac{r_{1}}{r_{2}}=\left(\frac{\varepsilon_{1}^{\prime}-i \varepsilon_{1}^{\prime \prime}}{\varepsilon_{2}^{\prime}-i \varepsilon_{2}^{\prime \prime}}\right)^{1 / 2}=\left(\frac{\varepsilon_{1}^{\prime}}{\varepsilon_{2}^{\prime}}\right)^{1 / 2}
$$

Following Lane and Saxton (1952), the dielectric constant of the sea water, $\varepsilon_{2}^{r}$ is given by

$$
\varepsilon_{2}^{r}=\varepsilon_{2}^{\prime}-i \varepsilon_{2}^{\prime \prime}
$$

where

$$
\begin{aligned}
& \varepsilon_{2}^{\prime}=\left[\varepsilon_{\infty}+\left(\varepsilon_{s}-\varepsilon_{\infty}\right) /\left(1+\omega^{2} \tau^{2}\right)\right], \\
& \varepsilon_{2}^{\prime \prime}=\left[\left(\varepsilon_{s}-\varepsilon_{\infty}\right) \omega \tau /\left(1+\omega^{2} \tau^{2}\right)+\sigma_{i} / \omega \varepsilon_{0}\right] .
\end{aligned}
$$

The different terms in (19) are given by Hollinger (1973). Here $T$ is the temperature of the sea water $\left(25^{\circ} \mathrm{C}\right)$ and $S$ the salinity $(35 \%)$. Troitsky $(1962)$ has given the following expression for the complex dielectric constant of foam water, consisting of air and sea water,

$$
\varepsilon_{1}^{r}=\varepsilon_{2}^{r}\left[1-\frac{3 R}{\left(2 \varepsilon_{2}^{r}+1\right) /\left(\varepsilon_{2}^{r}-1\right)+R}\right],
$$

where $R(=0.97)$, the volume ratio, is the ratio of volume of air in foam to the total volume of foam.

Using the above formulations, at $13.9 \mathrm{GHz}$, the frequency of radar altimeter, the plot 


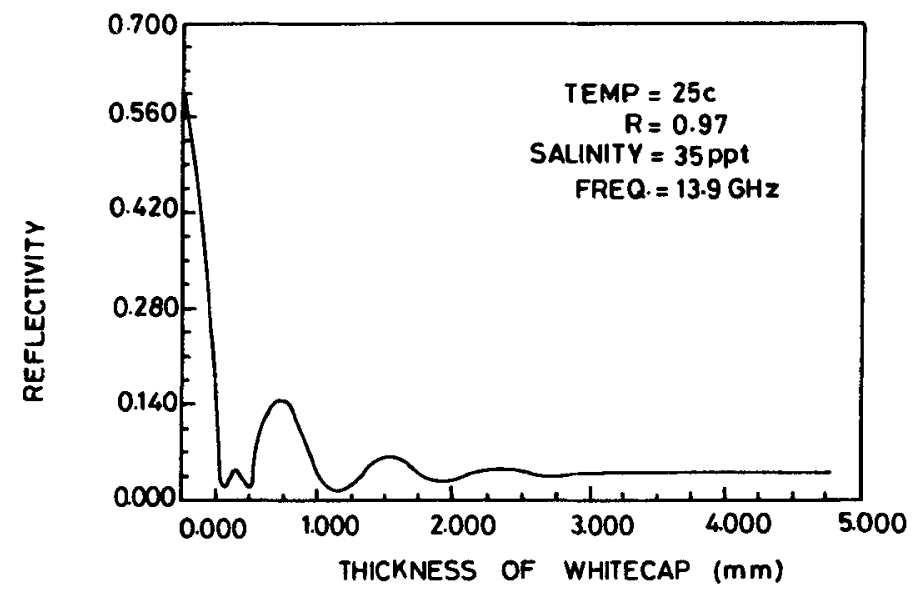

Figure 2. Microwave reflectivity versus thickness of whitecaps at $13.9 \mathrm{GHz}$.

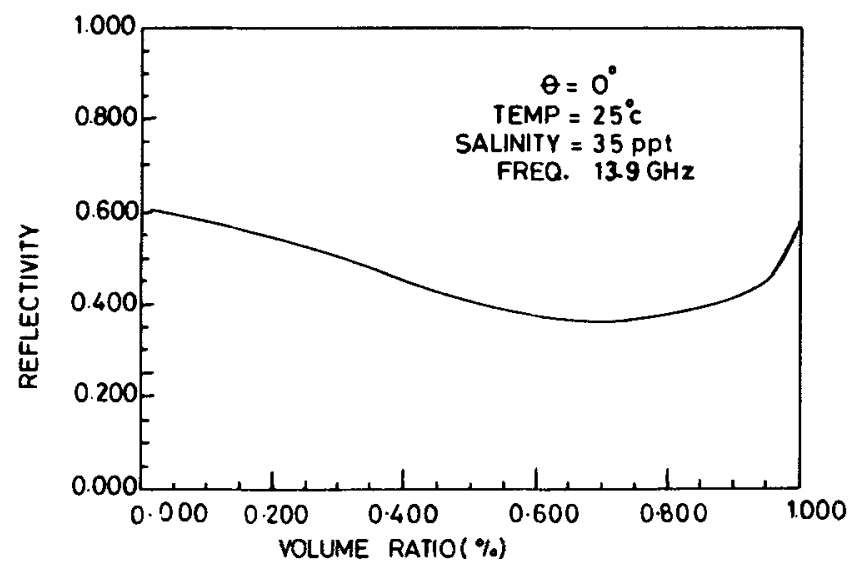

Figure 3. Effect of volume ratio $(R)$ on reflectivity.

of $r f$ versus $h l$, the thickness of the foam layer is given in figure 2. A change in reflectivity from a value of 0.6 to 0.1 is noticed for a foam thickness of $\sim 0.1 \mathrm{~cm}$. The oscillation in the curve is due to resonant absorption phenomenon in the foam layer.

Variation of reflectivity versus volume ratio has also been studied and depicted in figure 3.

4. Reflectivity calculations with an emissivity model and its comparison with the two-layer model

Several microwave emissivity models have recently been proposed by different workers (Wilheit 1979; Pandey and Kakar 1982) for interpreting data using passive microwave radiometers. Microwave emissivity is related to the reflectivity by the following 
relation,

$$
\text { emissivity }=1 \text { - reflectivity. }
$$

These relations were used to check the calculations presented in the preceding section using an empirical emissivity model proposed by Pandey and Kakar (1982). The emissivity is expressed as

$$
\varepsilon_{p}(\theta)=\left[\varepsilon_{s p}+\Delta \varepsilon_{r p}\right](1-F)+\varepsilon_{f p} F,
$$

where $p$ denotes polarization, which may be horizontal or vertical, $(\theta)$ is the incidence angle, $\varepsilon_{s p}$, specular emissivity and $\varepsilon_{r p}$ and $\varepsilon_{f_{p}}$ are contribution to emissivity due to roughness and foam for a case appearing to be the nadir,

$$
\begin{aligned}
& \varepsilon_{r p}=\frac{U_{10}}{T_{s}}(1.59)-0.00065 v, \\
& \varepsilon_{f p}=0.005 v+\frac{208+1.29 v}{T_{s}} .
\end{aligned}
$$

Where $v$ is the frequency in $\mathrm{GHz}$, the fractional foam coverage is given in terms of wind speed $U_{10}$, by the following expression

$$
F=a_{0}+a_{1} U_{10}+a_{2} U_{10}^{2} \text {. }
$$

The coefficients of (24) are given by

$$
\begin{aligned}
& a_{0}=1.707 \times 10^{-2}+8.560 \times 10^{-4} v+1.120 \times 10^{-5} v^{2}, \\
& a_{1}=1.501 \times 10^{-2}+1.821 \times 10^{-3} v-4.634 \times 10^{-5} v^{2},
\end{aligned}
$$

and

$$
a_{2}=2.442 \times 10^{-4}-2.282 \times 10^{-6} v+4.194 \times 10^{-7} v^{2} \text {. }
$$

We have compared reflectivity calculated through layered media model and through emissivity model calculations using a foam thickness of $0.1 \mathrm{~cm}$. The results for few cases are given in table 1.

\section{The effect of foam on wind speed estimation}

From (6), the backscattering coefficient for normal incidence angle is given by

$$
\sigma^{\circ}(0)=|R(0)|^{2} / \bar{S}^{2} \text {. }
$$

Table 1. Reflectivity through layered model vs. emissivity model.

\begin{tabular}{ccc}
$\begin{array}{c}\text { Wind speed } \\
(\mathrm{m} / \mathrm{sec})\end{array}$ & $\begin{array}{c}\text { Reflectivity through } \\
\text { a layered model } \\
(h=0-1 \mathrm{~cm})\end{array}$ & $\begin{array}{c}\text { Reflectivity through } \\
\text { an emissivity model }\end{array}$ \\
\hline 0.0 & 0.5916 & 0.5942 \\
5.0 & 0.5730 & 0.5773 \\
10.0 & 0.5669 & 0.5636 \\
15.0 & 0.5510 & 0.5499 \\
20.0 & 0.5171 & 0.5205 \\
25.0 & 0.4529 & 0.4507 \\
\hline
\end{tabular}


Following $W / u(1972), S^{2}$, the mean square slope, is given by

$$
\begin{aligned}
S^{2} & =a \ln U_{10}+b, \\
a & =0.010, \quad b=0.012 \ldots \ldots \ldots U_{10} \leqslant 7 \mathrm{~m} / \mathrm{sec}, \\
a & =0.850, \quad b=-0.145 \ldots \ldots U_{10} \geqslant 7 \mathrm{~m} / \mathrm{sec} .
\end{aligned}
$$

Equating (25) and (26) we have

$$
\sigma^{\circ}(\theta)=|R(0)|^{2} /\left(a \ln U_{10}+b\right) \text {. }
$$

Modifying the reflectivity in the presence of spray and foam, based on the previous analysis (\$4), (27) becomes

$$
\sigma^{\circ}=\left[|\mathbf{R}(0)|^{2}(1-F)+r_{1} F\right] /\left[a \ln U_{10}+b\right] \text {. }
$$

The model by Brown (1979) for wind speed measurement is given by

$$
\begin{aligned}
\sigma^{\circ}(\mathrm{d} B) & =-2.1-10 \log \left(A \ln U_{10}+B\right) \\
A & =0.02098, \quad B=0.01075 \ldots \ldots U_{10} \leqslant 9.2 \mathrm{~m} / \mathrm{sec}, \\
A & =0.08289, \quad B=-0.12664 \ldots \ldots U_{10} \geqslant 9.2 \mathrm{~m} / \mathrm{sec} .
\end{aligned}
$$

The $\sigma^{\circ}$ calculated from the modified expression (28) and Brown's model show better agreement at higher wind speed showing the importance of foam in the wind speed retrieval (figure 4).

A comparison of the wind speed derived using the present modified model with that of Brown's empirical model is shown in the figure (5) using Seasat-radar altimeter data in the Arabian sea and the Bay of Bengal. The data spanned the period 27-29 July and 4-11 August 1978 and covered the latitudes $-10^{\circ}$ to $+20^{\circ}$ and longitudes $60^{\circ}$ to $100^{\circ}$. The data over land was excluded from the analysis. The comparison gave an rms difference of $2 \cdot 2 \mathrm{~m} / \mathrm{sec}$. This was done since no concurrent ship observations were available for that period and region.

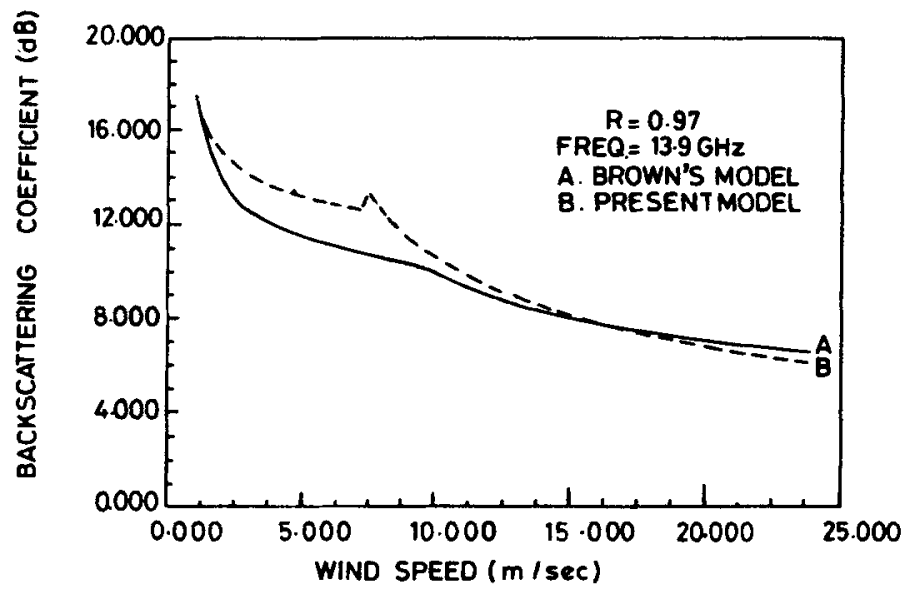

Figure 4. Comparison of $\sigma^{\circ}(\mathrm{d} B)$ versus wind speed $\left(U_{10}\right)$. 


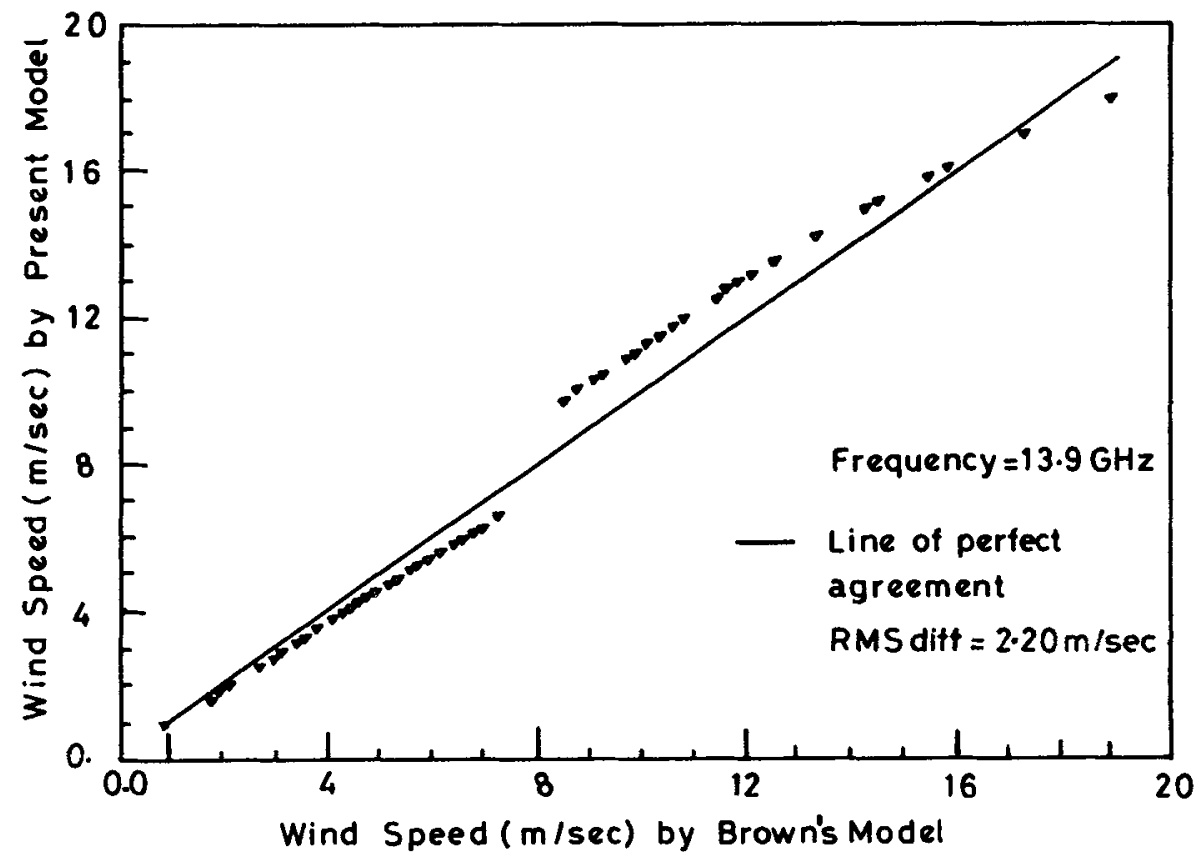

Figure 5. Comparison of altimeter inferred wind speeds by Brown's model and the present model.

\section{Conclusions}

The modified model proposed in the paper provides a better agreement with Brown's empirical model for wind speed retrieval. The agreement is more pronounced at higher wind speed. Seasat-radar altimeter data over the Arabian sea and the Bay of Bengal were used for wind speed extraction and a comparison with Brown's model gave an rms difference of $2 \cdot 20 \mathrm{~m} / \mathrm{sec}$.

\section{Acknowledgement}

We thank Dr T A Hariharan for encouragement. Comments and suggestions from Shri B S Gohil, S M Bhandari and Dr P S Desai, are gratefully acknowledged.

\section{References}

Barric D E 1974 IEEE Trans. Antennas Propag. AP-22 135

Brown G S 1978 IEEE Trans. Antennas Propag. AP-26 472

Brown G S 1979 J. Geophys. Res. 843974

Cox C S and Munk W H 1954 J. Opt. Soc. Am. 44384

Hollinger J P 1973 Aug 15, Microwave properties of the calm sea Rep. No. 7110-2 NRL, Washington DC 20375

Lane J A and Saxton J A 1952 Proc. R. Soc. London 214531

Mognard N M 1984 Proc. of an EsA Workshop held at Frascali, Italy, 8-11 May 
Pandey P C 1983 Linear retrieval and global measurements of wind speed from Seasat SMMR Publication no. 83-5, Jet Propulsion Laboratory, Pasadena, California

Pandey P C and R K Kakar 1982 IEEE Oceanic Eng. OE-7 135

Troitsky V S 1962 (eds) Z Kopal and H Mikhilov Radioemission of the moon, its physical state and the nature of its surface in the moon (New York: Academic Press)

Valenzuela G R 1978 Boundary-Layer Meteorol. 1361

Wait J R 1962 Electromagnetic waves in stratified media (New York: Macmillan)

Wilheit T T 1979 A model for the microwave emissivity of the ocean's surface as a function of wind speed NASA TM 80278, April

Wu J 1972 Phys. Fluids 15 741-746

Zheng Q A, Klemas G S, Hayne G S and Huang N E 1983 J. Geophys. Res. 38(C4) 2571 